\title{
Visual Analysis of Eye Gazes to Assist Strategic Planning in Computer Games
}

\author{
Ayush Kumar \\ Stony Brook University \\ aykumar@cs.stonybrook.edu
}

\author{
Michael Burch \\ TU Eindhoven \\ m.burch@tue.nl
}

\author{
Klaus Mueller \\ Stony Brook University \\ mueller@cs.stonybrook.edu
}

\begin{abstract}
This work studies the use of a conventional eye tracking system for analysis of an online game player's thinking processes. For this purpose, the eye gaze data of several users playing a simple online turn-based checkers game were recorded and made available in real-time to gaze-informed players. The motivation behind this work is to determine if making the eye-gaze data available can help these players to predict the gaze-tracked opponent player's further moves, and also how this can be most effectively done. We also tested different orientations of the screen on which the gaze data were displayed. By our visual and algorithmic analysis we validated (1) that prediction is possible and (2) that accuracy highly depends on the moves of players throughout the game as well as on the screen orientation. We believe that our study has implications on visual problem solving in general, especially in collaborative scenarios.
\end{abstract}

\section{CCS CONCEPTS}

- Computer systems organization $\rightarrow$ Embedded systems; Redundancy; Robotics; • Networks $\rightarrow$ Network reliability;

\section{KEYWORDS}

Eye-tracking, eye gazes, cognition, visualization, visual analytics, heat map

\section{ACM Reference Format:}

Ayush Kumar, Michael Burch, and Klaus Mueller. 2018. Visual Analysis of Eye Gazes to Assist Strategic Planning in Computer Games. In ETVIS'18: 3rd Workshop on Eye Tracking and Visualization, June 14-17, 2018, Warsaw, Poland. ACM, New York, NY, USA, 5 pages. https://doi.org/10.1145/3205929. 3205935

\section{INTRODUCTION}

Digital games have been around for more than half a century now, but eye movement and visual attention studies have piqued the interest of researchers much earlier than that and many techniques have been devised for this purpose [Almeida et al. 2011]. Studies have shown that eye gaze is one of the most reliable indicators of what a person is "thinking about" [Henderson and Ferreira 2013]. The direction of the gaze carries information about the focus of the

Permission to make digital or hard copies of all or part of this work for personal or classroom use is granted without fee provided that copies are not made or distributed for profit or commercial advantage and that copies bear this notice and the full citation on the first page. Copyrights for components of this work owned by others than ACM must be honored. Abstracting with credit is permitted. To copy otherwise, or republish, to post on servers or to redistribute to lists, requires prior specific permission and/or a fee. Request permissions from permissions@acm.org.

ETVIS'18, June 14-17, 2018, Warsaw, Poland

(C) 2018 Association for Computing Machinery.

ACM ISBN 978-1-4503-5787-6/18/06 ..\$15.00

https://doi.org/10.1145/3205929.3205935 user's attention [Just and Carpenter 1976]. Specifically in human cognitive processing tasks, the eye gaze is tightly linked to brain processing [Griffin and Bock 2000]. Additionally, directly before speaking a word or taking any action, the eyes move to the object about to be addressed [Griffin and Bock 2000] and the same could be related with any task that is to be performed. Not only is eye gaze highly reliable, it is also an implicit, subconscious reflex of the thinking process [Griffin and Bock 2000].

Thus far, the application of eye tracking in gaming and nongaming environments have mostly been used as a means for input, substituting or complementing traditional input methods such as a mouse, a keyboard or a joystick [Isokoski et al. 2009; Nacke et al. 2010; Sundstedt 2012]. The main focus of using eye tracking as an alternative input method is to make games more accessible to those with motor difficulties where eye movements can become an aid for game control [Špakov 2005].

While eye tracking has been well proven and demonstrated as a valuable analysis and evaluation instrument in areas such as the web [Almeida et al. 2010], metro maps [Kumar et al. 2018; Netzel et al. 2017] and television [Brasel and Gips 2008], it has yet to convince game researchers. Our work seeks to make advances in that direction.

Motivated by these findings, we have been investigating the role of eye gaze in human machine interaction. We selected a particular application to gain insight on how this might be accomplished, namely, the moves made by players while playing two-player or multi-player games. To reach this goal, we designed a visual analysis approach that is able to support the insight detection, i.e., we become able to derive patterns and anomalies from the eye movement visualization. We conducted our research with a controlled system where users can look at a graphical interface while playing the game, and at the same time are able to look into the gaze plots of the opponent making it more or less a real-time visual analysis application. It is different from systems in which users are not proactively involved. In contrast, eye gaze in the proposed work is a subconscious input which naturally occurs within the cognitive process. Since eye movements are executed automatically and nonvoluntarily, this unique setting is fundamental for understanding any human interaction with graphical interfaces.

This paper reports on our approach on this topic primarily addressing the object activation problem as well as first results we have obtained. The second part of this paper addresses work related to game and gaming environments with eye tracking. Section 3 describes the complete experimental set-up as well as the way in which the experiment has been conducted. The fourth section summarizes the results and the conclusion along with future work is discussed in the fifth section. 


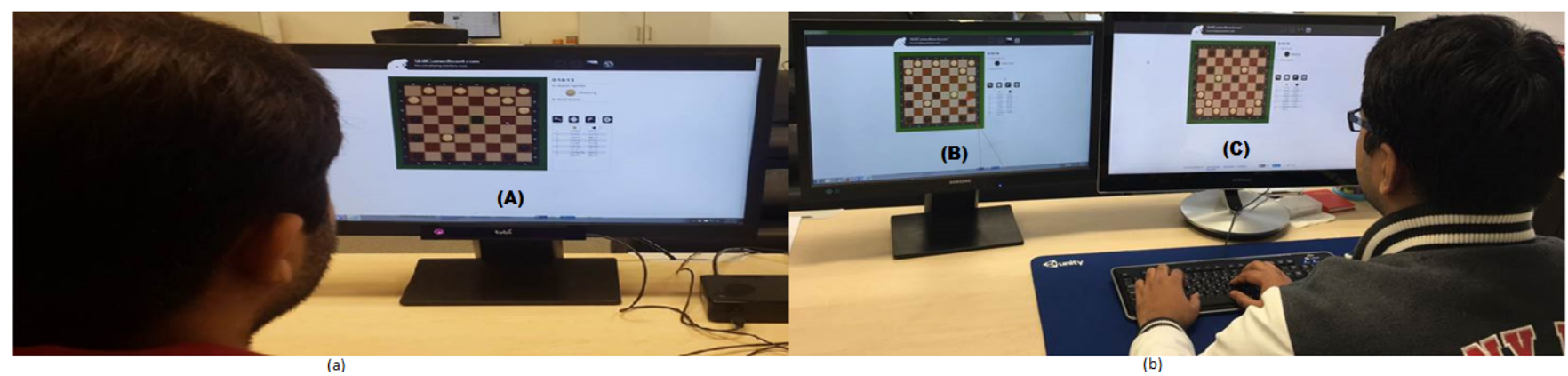

Figure 1: (a) : Display (A) augmented with an eye tracker used by the first player - the gaze-tracked player (GTP). (b) : Auxiliary Display (B) with eye gaze plots derived from the GTP's eye gazes to help the second player - the gaze-informed player (GIP) in predicting GTP's moves on Display (C) and making his own moves on (C). The two players sit in well separated locations.

\section{RELATED WORK}

Isokoski et al. [Isokoski et al. 2009] identified four different ways in which eye tracking could be implemented in games. The first does not require any specific modification within the game and is known as the 'dwell-time based selection' technique. The second solution requires the use of additional software. The third solution implies modifications in the game code which will allow eye control. The fourth solution is the most labor intensive and implies the development of a game from scratch.

More similar to our work, Patrick et al. [Jermann et al. 2010] used eye gazes for post-analysis to unveil the coordination and expertise of players in collaborative Tetris. However, they did not use eye gazes for collaboration tasks in real time. They note that real-time feedback can be quite disturbing because it interferes with the main task representation and because it is not always precise enough to support deictic references. Various other systems, such as Clearboard [Ishii and Kobayashi 1992] or GAZE groupware [Vertegaal 1999] have also been designed to give collaborators access to the gaze directions of others, similarly to a face-to-face situation. Charness et al. [Charness et al. 2001] also used eye tracking to find the perceptual aspect of skilled performance in chess and found the differences in attention allocations between experts and novices. Küchelmann et al. [Küchelmann et al. 2017] have introduced an efficient way for automatic analysis of chess player's visual behavior that can be used for setting up an intuitive chess assistant system for training purposes.

To the best of our knowledge, to date no commercial games have been developed to make full use of eye tracking. However, many individual and academic projects have resulted in original or adapted games that explore the potential of eye tracking as an input method. We specifically address the genre of basic strategic computer games where turn based moves make it easier to access the data and make it less challenging as described in [Jermann et al. 2010].

\section{EYE TRACKING STUDIES}

Our two-player experimental setup is heterogeneous in which only one player has access to the eye-gazes of the other player. This allows us to assess the advantages this first player has over the second, if any. We call the first player the GIP - the gaze-informed player while we call the second player the GTP - the gaze-tracked player. Our study focuses on the eye-and-mind relation in the gaming environment, where the GIP might be able to predict the GTPs move before he actually makes it. We hypothesize that this methodology will yield insight in building a multi-player gaze-based gaming platform where displaying a players eye gazes might lead to better communication among several players in a team. To find insights into the visual task solution strategies and design interface, of the study participants we compare their eye movements as dependent variables recorded during the eye tracking studies.

\subsection{Hypothesis \& Research Question}

The main purpose of this work is to study eye behavior in a gaming environment, namely a turn based multi-player game, specifically on how players direct their gaze, what they fixate at, where they fixate, and when they fixate certain things. We consider eye fixations as measurements of the player's ongoing state of mind and evolving strategy in solving the problem at hand, i.e. winning the game by making these gazes available to the opponent (the GIP) to predict the player's moves (the GTP). As stated in [Jermann et al. 2010], real-time feedback can be quite disturbing, and so a strategic game was chosen which gives enough time to analyze the gazes gathered from one player and provided as feedback for the other. This arrangement was expected to give positive results for the player who has access to eye gazes in order to predict the opponent's move.

Fig. 1 shows the physical setup. The GTP's and GIP's primary displays are (A) and (C), respectively. Having read much of the literature on the eye-mind relation [Just and Carpenter 1976], that a tight coupling exists between eye and the mind, which lays the groundwork for our hypothesis H1 : we expect that it would not be difficult for the GIP to predict the next moves of the GTP, when GTP gaze data was available on (B) as real-time feedback. We tested two orientations for Auxiliary Display (B) on which the GIP has access to the GTP's eye gazes: (1) NORMAL (Figure 2(a)) where the display is aligned with the GTP's primary screen (A), and (2) with a $180^{\circ}$ rotation, aligned with the GIP's primary screen $(\mathrm{C})$ (Figure 2(b)). H2 : We expect that the GIP will be able to do better predictions with orientation (2). To prove this hypothesis, we aimed 
to find out how accurately GIP can predict GTP fixation-based next moves and draw conclusions on intent.

\subsection{Stimuli and Task}

We used an online checkers game for this experiment. Our game design offers four alternative moves, i.e. forward diagonal left \& right and backward diagonal left \& right, unlike other checker games with only two alternative moves i.e. diagonal left and right only in the forward direction. The objective of the game is to get as high a score as possible and predict all of the GTP's moves accurately. All pieces on the checkerboard are visible to both players at all times. As the game proceeds, the GIP was tasked to predict the moves of the GTP (based on the eye gazes available to him) before the GTP makes the move (but is ready to move). The prediction includes: (1) the piece the GTP will move and (2) the place where the piece will be moved. In our experiment, GTP is asked to think aloud, i.e. has to tell the GIP that he was ready for making a move. This was done to let GIP know that GTP is decided with his move and GIP can predict the same, which is validated by the experimenter.

\subsection{Environment Condition and Technical Setup}

The study was conducted in a laboratory isolated from outside distractions. Participants were instructed to switch off their mobile phones to reduce possible distractions during the study. A Tobii X2-60 eye tracker was associated with Display (A) for the GTP. It was linked to the Auxiliary Display (B) for the GIP who used Display (C) to view the actual moves of the GTP and make his own (refer to Figure 1). During the experiments, the GTP was asked to sit in front of (A) with the eye tracker fixed on him at a distance of about 60-65 cm (Figure 1a). Conversely, the GIP could not see (A) (and vice versa) but could view the GTP's eye-trajectories on (B) (Figure 1b). A standard 9 point calibration was done before each study for calibrating eye tracker.

\subsection{Participants}

The study participants were students from our university. We chose a between subject study design with 18 participants which resulted into 9 study trials. All participants reported that they read text from left to right and were right handed. We did not specifically test for color blindness as it was deemed irrelevant since color plays a minor role in our experiment. There were 8 females and 10 males. Their average age was 23 years; the youngest participant was at the age 19 and the oldest at 31 . All participants had normal or correctedto-normal color vision and three of them wore glasses while two of them wore contact lenses. However since it proved difficult for the eye tracker to detect the eye gazes of participants with contact lenses we did not take their plots into consideration. Only 2 out of 18 reported that they had played checkers before.

\subsection{Study Procedure}

This experiment was conducted in two set-ups, where a GTP was asked to play the game and was asked to sit away from Display (B) and (C) which were placed together so that the GIP could easily view the gaze plots on (B) (Figure 1). The set-up for the first experiment was done with similar orientations of the screens of (A) and (B) as

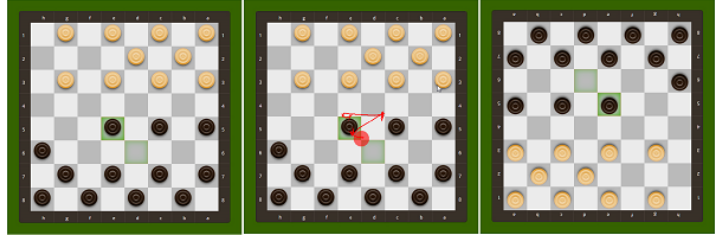

(a)

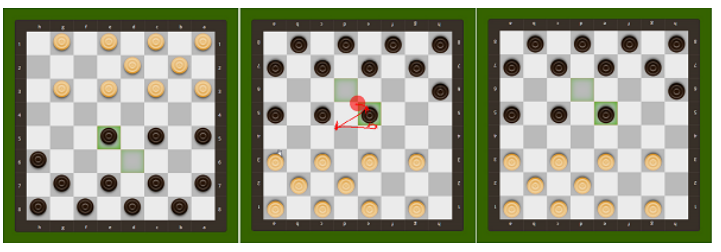

(b)

Figure 2: (left to right) Display A augmented with an eye tracker (control PC) used by the GTP, (center) Auxiliary Display B with eye gaze plots from GTP (similar to Display A) to help GIP moving the game pieces on Display C (shown on the right), with (a) being the NORMAL orientation of Display B, as of Display A, while (b) being rotated by $180^{\circ}$ to have orientation similar with the screen Display $C$.

shown in Figure 2(a) while the second set-up includes a rotation of (B) by $180^{\circ}$ to make it similar with the screen of (C) as shown in Figure 2(b).

The objective of the game for the GTP was to get as high a score as possible. A similar goal applies to the GIP with the additional task to predict the moves of the GTP. Each game was assumed to last five to seven minutes, with a brief period for synchronization at the start and end of the game from which 8 predicted moves were recorded by the experimenter for analysis of predicting accuracy at the end.

All participants played the game after a training phase for 2-3 minutes. The experiment involved two players where the GTP was playing the game normally against the GIP, whereas the GIP also had to track the gaze plot of the GTP through the Auxiliary Display PC (B). This was done by a special feature of the software Tobii Studio Live Viewer which let eye gazes to be viewed on another Display PC connected to the same system from which the control PC is being connected. The selection of GTP and GIP among 2 players were done randomly and their role was same throughout the study. The move by GTP was to be predicted by GIP and at the same time the GIP was asked to plan his move from the real-time analysis of GTP's eye gaze.

Three sets of data were recorded for each trial: (1) the eye tracker data, (2) the data related to the prediction which was recorded by the experimenter for 8 moves of both players, and (3) a simple questionnaire at the end. Eye movement data was recorded every $4 \mathrm{~ms}$ by the software. The prediction data resulted in 8 different correct or incorrect responses by the GIP. Data collected by the eye tracker during the experiment has many attributes but we were only interested in the eye gaze positions in both $\mathrm{x}$ and $\mathrm{y}$ space, the fixation duration which tells about the time one spent 
Table 1: Screen orientation effect (out of 8 moves on average)

\begin{tabular}{cccc} 
Orientation & Attempted & Correct & Correct $(\%)$ \\
\hline Normal & 8 & 5.5 & 68.75 \\
Inverted & 8 & 6.2 & 77.5
\end{tabular}

Table 2: Scanning board while playing (out of 8 moves on average)

\begin{tabular}{cccc} 
Scanning & Attempted & Correct & Correct $(\%)$ \\
\hline Partial & 8 & 5.9 & 73.75 \\
Full & 8 & 6.5 & 81.25
\end{tabular}

at a particular location, the saccade amplitudes, and the fixation index.The questionnaire consisted of six questions which dealt with participant expertise levels, their interest in online gaming and their experience while playing in both situations and rating them.

\section{RESULTS}

We witnessed that the orientation of views shown on (B) had a major role in helping the GIP in the prediction task. When the screen was in normal orientation (Figure 2(a)), the prediction accuracy was $68.75 \%$ as shown in Table 1 . This was due to the fact that it was difficult for the GIP to mentally switch the board orientation easily from (B) to (C), respectively, which is answered by the player's in the questionnaire answered at the end of the game. Changing the view orientation of (B) to match the board orientation of (C) as shown in Figure 2(b), boosted the prediction accuracy to $77.5 \%$ (see Table 1). This proves the hypothesis that screen orientation has a major impact on playing while making availability of eye gazes.

It can also be seen in Table 2, that prediction accuracy is $81.25 \%$ when the GTP scanned almost the whole board while playing, as compared to the scenario where only partial scanning (a section of checkerboard is covered) was done while playing, as shown in Figure 4 ( $2^{\text {nd }}$ row of $3 \times 3$ clustered gaze and heat map). By doing so the player likely tried to form a strategy to win and the eye gaze revealed this thinking process due to long scanpaths. However, this confirmed the hypothesis of the participant answers in the questionnaire, that it was easier for them to predict when there were less dots on the board resulting into larger movements by the eyes, which resulted in an overall scanning of the gaming board.

Another interesting finding is that the corners of the board has been neglected in most of the cases which can be seen in Figure 3 and Figure 4. This could be due to expertise level of players, calibration error or some other psychological phenomenon, which is yet to be proved by this study.

\section{CONCLUSION AND FUTURE WORK}

In this paper, we reported on an experiment where a board game player was informed by an opponent player's eye gazes while planning the next move. We used a computer (online) version of the game of checkers as the game environment. We observed that the eye gazes can indeed be used for prediction tasks and seem to have a relation with the thinking process of a player. We also found that it is advantageous to transform the gaze-tracked player's view

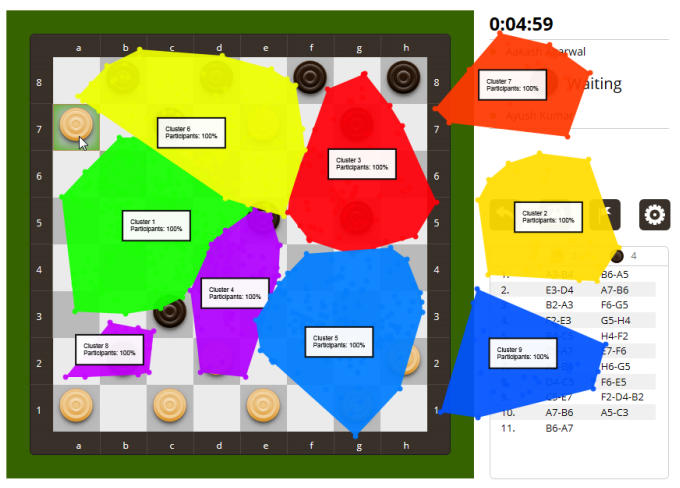

Figure 3: Clustered gazes where the left most lower part has been neglected whereas the whole of the board has been scanned during the game making larger saccades.

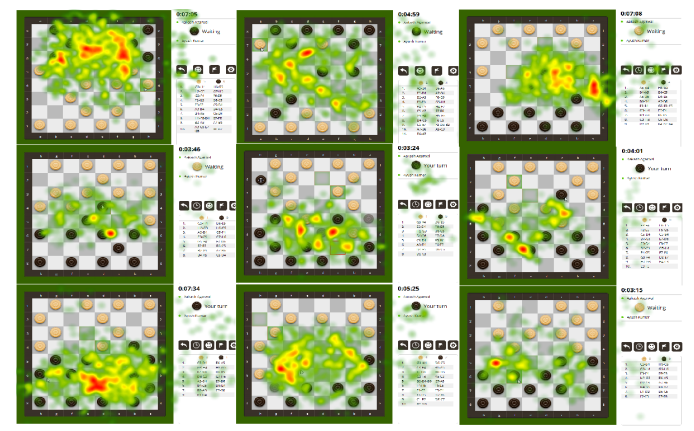

Figure 4: Heat map of gazes that reveal that the left lower part has been neglected in almost all of the test cases.

into that of the gaze-informed player. In our experiment, we chose a heterogeneous setup, but the same experimental methodology could also be used with both of the players having access to the opponent gazes.

We are currently investigating set-ups in which we provide the GIP information of the GTP's eye gazes for the last few seconds or for the entire last move in a more detailed manner, to determine if this will help the GIP. In this case, it might be that overlaying gazes for longer durations leads to visual clutter. Addressing this potential is the focus of this current work. What we also have not analyzed yet are the winning probabilities for the player with and without access to the opponent's eye gazes. This will also be addressed in our future work.

We believe that our research has promising implications in collaborative gaming with multi-players where gazes of respective teams could be used to design better strategies for the game. It might also have implications for collaborative work in general.

\section{ACKNOWLEDGMENTS}

This research was supported by NSF grant IIS 1527200 \& MSIT (Ministry of Science and ICT), Korea, under the ICT Consilience Creative Program (IITP-2017-R0346-16-1007) supervised by the IITP (Institute for Information \& Communications Technology Promotion). 


\section{REFERENCES}

Samuel Almeida, Óscar Mealha, and Ana Veloso. 2010. Interaction Behavior of Hardcore and Inexperienced Players:âĂIJCall of Duty: Modern WarfareâĂİ Context. (2010).

Samuel Almeida, Ana Veloso, Licínio Roque, and O Mealha. 2011. The eyes and games: A survey of visual attention and eye tracking input in video games. Proceedings of the SBGames, Salvador BA, Brazil 79 (2011), 110.

$S$ Adam Brasel and James Gips. 2008. Points of view: Where do we look when we watch TV? Perception 37, 12 (2008), 1890-1894

Neil Charness, Eyal M. Reingold, Marc Pomplun, and Dave M. Stampe. 2001. The perceptual aspect of skilled performance in chess: Evidence from eye movements. Memory \& Cognition 29, 8 (01 Dec 2001), 1146-1152. https://doi.org/10.3758/ BF03206384

Zenzi M Griffin and Kathryn Bock. 2000. What the eyes say about speaking. Psychological science 11,4 (2000), 274-279.

John Henderson and Fernanda Ferreira. 2013. The interface of language, vision, and action: Eye movements and the visual world. Psychology Press.

Hiroshi Ishii and Minoru Kobayashi. 1992. ClearBoard: a seamless medium for shared drawing and conversation with eye contact. In Proceedings of the SIGCHI conference on Human factors in computing systems. ACM, 525-532.

Poika Isokoski, Markus Joos, Oleg Spakov, and Benoît Martin. 2009. Gaze controlled games. Universal Access in the Information Society 8, 4 (2009), 323-337.

Patrick Jermann, Marc-Antoine Nüssli, and Weifeng Li. 2010. Using dual eye-tracking to unveil coordination and expertise in collaborative Tetris. In Proceedings of the 24th BCS Interaction Specialist Group Conference. British Computer Society, 36-44.

Marcel Adam Just and Patricia A Carpenter. 1976. Eye fixations and cognitive processes. Cognitive psychology 8, 4 (1976), 441-480.

Thomas Küchelmann, Paola Carolina Torche, Manjunath Prasad, Kai Essig, and Thomas Schack. 2017. Automatic Analysis of Players Behavior in Real Dyadic Chess Situations. In 1st Workshop on "Behavior, Emotion and Representation: Building Blocks of Interaction”. Bielefeld, Germany. https://hal.inria.fr/hal-01617691

Ayush Kumar, Rudolf Netzel, Michael Burch, Daniel Weiskopf, and Klaus Mueller. 2018. Visual Multi-Metric Grouping of Eye-Tracking Data. Journal of Eye Movement Research 10, 5 (2018). https://bop.unibe.ch/JEMR/article/view/4225

Lennart E Nacke, Sophie Stellmach, Dennis Sasse, and Craig A Lindley. 2010. Gameplay experience in a gaze interaction game. arXiv preprint arXiv:1004.0259 (2010).

Rudolf Netzel, Bettina Ohlhausen, Kuno Kurzhals, Robin Woods, Michael Burch and Daniel Weiskopf. 2017. User performance and reading strategies for metro maps: An eye tracking study. Spatial Cognition \& Computation 17, 1-2 (2017), 39-64. https://doi.org/10.1080/13875868.2016.1226839 arXiv:https://doi.org/10.1080/13875868.2016.1226839

Oleg Špakov. 2005. EyeChess: the tutoring game with visual attentive interface. Alternative Access: Feelings \& Games (2005), 81-86.

Veronica Sundstedt. 2012. Gazing at games: An introduction to eye tracking control. Synthesis Lectures on Computer Graphics and Animation 5, 1 (2012), 1-113.

Roel Vertegaal. 1999. The GAZE groupware system: mediating joint attention in multiparty communication and collaboration. In Proceedings of the SIGCHI conference on Human Factors in Computing Systems. ACM, 294-301. 